\title{
THE SIGNIFICANCE OF PROTEIN AND NON-PROTEIN TUBERCULIN SENSITIVITY AND INSENSITIVITY IN SARCOIDOSIS
}

\author{
William Hartston, M.D., M.R.C.P., D.T.M., D.P.H. \\ Dorothy Temple Cross Research Fellow, Medical Research Council, from the Chest Clinic, St. Thomas' Hospital and \\ the Skin Department, London Hospital
}

ONE of the more perplexing features of sarcoidosis is the abnormally high rate of insensitivity to tuberculin injected intradermally. In any large urban community in England very few adults between ages 30 and 65 will be found not reacting to a dose of 100 units ( $0.002 \mathrm{mg}$.) of tuberculin; fewer still will be found in a comparable age-group of ambulant cases of tuberculosis, yet half or more of a group of cases of active sarcoidosis may be found to be insensitive to roo units of Purified Protein Derivative (PPD) tuberculin.

The significance of this 'anergic' state in patients showing lesions with sarcoid histology, is fundamental to understanding the immunological abnormality in this condition.

\section{Implications of Cutaneous Tuberculin Reactivity}

It is usually assumed that the protein element in the tubercle bacillus is the agent responsible for stimulating cutaneous tuberculin reactions. Hartston and Muggleton (I962) have shown that other and non-protein fractions are more closely and specifically associated with tuberculin sensitivity. They demonstrated reactivity in 'normal' and tuberculous subjects, to a lipopolysaccharide fraction of human-type tubercle bacillus and that following BCG vaccination, there developed a spontaneous local skin reaction at the site of the previous prevaccinal non-reacting injections. Similar studies have been made in cases of sarcoidosis. The findings are reported here and their significance is discussed.

Tuberculin allergy depends for its pathogenesis on an immunological reaction taking place in the affected tissues between the provocative antigen and antibody. When mycobacteria such as tubercle bacilli or BCG invade the body, their chemical constituents are incorporated into the protoplasm of capillary perithelial cells, connective tissue cells, epithelial cells, reticuloendothelial cells and lymphocytes which thereby become sensitized to the incorporated chemical substances, provoke antibodies to them and mank fest hypersensitivity reactions when they recognize this alien bacterial antigen reintroduced. Once the chemical union has taken place and bees 'registered' in the cells, it is no longer essential fóp living bacilli to subsist in the body to maintait tuberculin hypersensitivity. By this mechanism the affected tissue cells accentuate their capacit to hydrolyze bacterial walls, meet their cytoplasing and generate the factors which create and transfer tuberculin allergy. This response is developed to its highest degree under the influence of mygobacterial lipopolysaccharides.

When the bacterial wall is readily breached find sensitivity reactions rapidly follow, these maylifs as damaging to the host as to the bacterium, as if caseating tuberculosis. When the invading bace terial wall for any reason is unaffected or only verw slowly disintegrated, then a cellular granulomatous reaction is more likely to ensue as happens it sarcoidosis and leprosy.

\section{Tuberculin Insensitivity}

These responses may be inhibited by de्s sensitizing with repeated subcutaneous doses tuberculin or by some other functional modificas tion of the antibody-forming mechanism such as interference with the normal activity of lympho? cytes. Although tuberculin densensitization abolishes the local (skin) responses to tuberculin it does not impair immunity already acquired.

Failure to react to tuberculin occurs in those. who have never met adequate amounts of mycof bacteria to stimulate the response, or in infected people in whom the immunity mechanism hr failed.

The vascular constituent of the tuberculio reaction but not the cellular infiltrate can be inhibited by direct suggestion under hypnos晴 (Black, Humphrey and Niven, 1963). Advanced active tuberculosis and other conditions in which suprarenal deficiency occurs may be accompanie by suppression of tuberculin sensitivity. 
In sarcoidosis, absence of cutaneous response to tuberculin is more likely to occur when the condition is active, that is, when non-caseating epithelioid-cell follicles can be demonstrated in biopsies of tissue and sarcoid deposits are manifest. This anergic state may persist (Scadding, I960) even when cases of sarcoidosis develop pulmonary tuberculosis with bacilli in the sputum.

Patients whose tuberculin-negative sarcoidosis follows an episode of typical tuberculosis may also have been anergic during their tuberculous activity.

The frequency of the non-reactor state in sarcoidosis is clearly an important pathological condition which poses certain questions. Is it better for the body in sarcoidosis to be able to respond to tuberculin or better that it should maintain an immunological tolerance to chemical fractions of mycobacteria? Which part of the mechanism of adult tuberculin sensitivity is at fault ? Is it a failure of the tissue cells at the test site to recognize or to respond to the tuberculin antigen ?' Is there no antibody circulating or has the antibody produced been diverted from the skin and got fixed elsewhere? Are the lymphocyte cells abnormal ?

Sarcoidosis patients not reacting to tuberculin can in most cases be 'converted' by adequate BCG vaccine given by multipuncture or by intradermal injection. In the author's series of 62 cases ( 34 'active' and 28 'quiescent' or old) 21 were nonreactors to tuberculin both protein and nonprotein. Seventeen of these were given BCG vaccine and among these, 14 thereafter reacted to PPD but not to non-protein lipopolysaccharide. The 4I tuberculin-sensitive sarcoidosis cases reacted to both PPD and lipopolysaccharide. The significance of the sarcoid cases' failure to react to lipopolysaccharide fraction of human type $M$. tuberculosis after BCG vaccination is surprising and difficult to explain. It is possible that in these circumstances the sarcoid patient can make antibody to the protein fraction of mycobacteria but not to the lipid and polysaccharide.

\section{Discussion}

A group of five patients (not sarcoid) with active pulmonary tuberculosis, whose protein tuberculin sensitivity had been abolished by desensitizing with a prolonged course of subcutaneous tubercle bacillus extracts continued to react to lipopolysaccharide. Sarcoidosis patients who do not react to protein or non-protein tuberculins will become reactors to both after a subcutaneous 'donation' of leukocytes from a known normal tuberculin reactor.

It seems therefore that the antibody-forming mechanism can be stirred to activity, but the readiness of 'indigenous' lymphocytes to change to epithelioid cells and be diverted from normal activity makes them suspect as the weak link in the sarcoid's chain of immunological rectitude.

Converting the non-reacting sarcoid patient to tuberculin hypersensitivity appeared to have no beneficial effect on the sarcoidosis lesions except in one patient who developed bouts of erythema nodosum on both legs soon after BCG vaccination. During the healing of one crop of these red nodes a long-standing sarcoid skin lesion on one calf quickly disappeared and has not returned, although long-standing lung lesions have persisted.

One patient with widespread sarcoid nodules on the skin of his face and in both axillæ and who reacted to protein and non-protein tuberculin, was desensitized with subcutaneous injections of PPD and lipopolysaccharide. After seven weeks of this he failed to react to 750 units of intradermal PPD and to 50 micrograms of lipopolysaccharide and the facial and axillary lesions rapidly paled and shrank away. In this case at all events, there is no doubt of the benefits of not reacting to tuberculin.

There is another hint of advantage to the sarcoid patient in a reduced tuberculin sensitivity. Women with sarcoidosis who become pregnant tend to improve during the late months and early puerperium and to relapse afterwards. It is a common finding too, that tuberculin-reacting normal women develop much weaker tuberculin sensitivity during pregnancy.

\section{Summary}

The factors influencing tuberculin sensitivity and insensitivity are described. Their condition in sarcoidosis is discussed. It is suggested that the sarcoid patient who reacts to tuberculin might benefit from desensitizing and conversion to a non-reactor state.

I am indebted to Glaxo Research Laboratories for supplies of antigen and to Dr. John Anderson, Dr. N. Lloyd Rusby and Dr. Brian Russell for generous clinical facilities.

\section{REFERENCES}

Hartston, W., and Muggleton, P. W. (1962): Biological Activity of a Non-protein Tuberculin, Trans. Health and TB conf., p. 55. Ibadan, Nigeria.

BLACK, S., HuMPHREY, J. H., and Niven, J. S. (1963): Inhibition of Mantoux Reaction by Direct Suggestion under Hypnosis, Brit. med. Y., i, 1649.

ScadDING, J. G. (1960): Mycobacterium Tuberculosis in the Ætiology of Sarcoidosis, Ibid, ii, I618. 